\title{
Multi-fidelity fluid-structure interaction analysis of a membrane blade concept in non-rotating, uniform flow condition
}

\author{
Mehran Saeedi, Kai-Uwe Bletzinger, and Roland Wüchner \\ Lehrstuhl für Statik, Technical University of Munich, 80333 Munich, Germany \\ Correspondence to: Mehran Saeedi (mehran.saeedi@tum.de) \\ Received: 16 March 2016 - Published in Wind Energ. Sci. Discuss.: 11 May 2016 \\ Revised: 28 September 2016 - Accepted: 28 October 2016 - Published: 23 November 2016
}

\begin{abstract}
In order to study the aerodynamic performance of a semi-flexible membrane blade, fluid-structure interaction simulations have been performed for a non-rotating blade under steady inflow condition. The studied concept blade has a length of about $5 \mathrm{~m}$. It consists of a rigid mast at the leading edge, ribs along the blade, tensioned edge cables at the trailing edge and membranes forming the upper and lower surface of the blade. Equilibrium shape of membrane structures in the absence of external loading depends on the location of the supports and the prestresses in the membranes and the supporting edge cables. Form-finding analysis is used to find the equilibrium shape. The exact form of a membrane structure for the service conditions depends on the internal forces and also on the external loads, which in turn depend on the actual shape. As a result, two-way coupled fluid-structure interaction (FSI) analysis is necessary to study this class of structures. The fluid problem has been modelled using two different approaches, which are the vortex panel method and the numerical solution of the Navier-Stokes equations. Nonlinear analysis of the structural problem is performed using the finite-element method. The goal of the current study is twofold: first, to make a comparison between the converged FSI results obtained from the two different methods to solve the fluid problem. This investigation is a prerequisite for the development of an efficient and accurate multi-fidelity simulation concept for different design stages of the flexible blade. The second goal is to study the aerodynamic performance of the membrane blade in terms of lift and drag coefficient as well as lift-to-drag ratio and to compare them with those of the equivalent conventional rigid blade. The blade configuration from the NASA-Ames Phase VI rotor is taken as the baseline rigid-blade configuration. The studied membrane blade shows a higher lift curve slope and higher lift-to-drag ratio compared with the rigid blade.
\end{abstract}

\section{Introduction}

Flexible wings have been the topic of many research programs. Different techniques have been used in order to bring flexibility to conventional wing configurations. They range from using new structural concepts for wing frames like telescopic spars (Blondeau et al., 2003) or morphing wings (Bowmann et al., 2007), to using smart materials in the manufacturing of the wing (Barbarino, 2010). Whereas in active control concepts (like morphing wings), deformability is brought to the wing by the use of actuators, in passive control the wing is to some extent flexible and is deformed solely as a consequence of applied aerodynamic loads. In the case of passive control the final form of the wing is a result of the equilibrium between aerodynamic forces and internal structural forces, and therefore it is not trivial to reach the desired final shape. With the increase in a wind turbine's rotor diameter, aeroelastic simulation of rotor blades to study their unsteady response to disturbances or control actions has become more and more important. To realize the so-called "smart rotors" both active (Barlas et al., 2016) and passive (Bottasso et al., 2016) aeroelastic devices have been studied for load mitigation of wind turbines. 
Membrane wings have proved to be a good alternative to rigid wing constructions for micro air vehicles (by definition with a maximum dimension of $15 \mathrm{~cm}$ ) (Lian and Shyy, 2005; Abdulrahim et al., 2005). The flexibility of a membrane wing enables it to adapt itself to the flow field to a certain extent. The advantages of this passive adaptation to the surrounding flow are, from an aerodynamics point of view, a higher lift slope, higher maximum lift coefficient, and a postponed stall to higher angles of attack compared to rigid wings (Valasek, 2012) and, from the structural perspective, load reduction in unsteady-flow cases (Levin and Shyy, 2001). One drawback of flexible wings could be that because of their flexibility and due to self-excited vibrations they could show an unsteady response even to steady-flow conditions (Waszak et al., 2001).

Specific sailwing research was originally initiated at Princeton University during the 1970s with an interest in determining the applicability of this design as an auxiliary lifting device. Various studies were employed to explore the structural and aerodynamic characteristics of the sailwing. It was concluded that sailwings have favourable characteristics compared with conventional rigid wings. From an aerodynamics point of view, sailwings have a higher lift curve slope, a higher maximum lift coefficient, and a higher liftto-drag ratio compared to an equivalent rigid wing (Fink, 1967, 1969; Maughmer, 1979; Saeedi et al., 2015). Delayed stall to higher angles of attack is another advantage of membrane wings (Maughmer, 1979). From a structural dynamics point of view, there is a load reduction for membrane wings in unsteady-flow cases (Levin and Shyy, 2001). Later, during the 1980s, the application of the sailwing concept to wind energy systems was explored by the Princeton windmill group. The final progress report of the group states (Maughmer, 1976) that "the sailwing rotor continues to be highly competitive in performance with its rigid-bladed counterparts and yet enjoys the benefits of simpler construction and lower costs".

The membrane blade concept studied in the current work is mostly a passively controlled wing. Membrane structures are able to efficiently carry external loads over large spans via internal in-plane stresses. This optimal load-carrying behaviour is inherently accompanied by a significant flexibility. The structural response of such a wing to aerodynamic loads depends on the membrane's stresses, so two-way coupled fluid-structure interaction simulations (FSI) are necessary to analyse its behaviour in operation. Different techniques are used to model FSI problems. One possibility is to use separate meshes allowing field-specific resolutions for the fluid and structural domain, with a body-fitted mesh at the coupling interface for the fluid domain. The arbitrary Lagrangian-Eulerian (ALE) formulation is typically used in this method and the fluid mesh needs to be updated after each iteration. In principle, updating could be done by either remeshing of the fluid domain or by applying the displacement at the FSI interface to the original mesh and recalculating the nodal coordinates. An alternative method is using the embedded method (Viré et al., 2015). In this approach an extended mesh is used for both the fluid and the solid domain. The actual position of the interface between the two domains is represented in the extended mesh and the presence of the solid and its effect on the fluid domain is represented as an additional source term in the momentum equation. The method uses the Eulerian method for the fluid domain, while the solid part is represented in a Lagrangian manner. As an alternative approach, the moving-fluid interface could also define the structure domain which is cut out of the fluid mesh with suitable boundary conditions (Baumgärtner et al., 2015).

Numerous aeroelastic simulations might be needed to find the best set of prestresses and material parameters to ensure a better aerodynamic performance. This highlights the need for less complex (and thus computationally less expensive) fluid models for FSI simulations during early design stages. An alternative to the numerical simulation of the flow field using Navier-Stokes equations (NSE) is the vortex panel method. The panel method is computationally less demanding and enables a faster exploration of the design space. However, in general, it neglects viscous effects and therefore its range of applicability should be evaluated. In the current paper FSI simulations for the membrane blade concept have been performed using these two approaches for the fluid side and the results are systematically compared. Aerodynamic performance of the membrane blade is checked against its equivalent rigid blade as well.

Analysis of the membrane blade consists of three major steps. They are represented in Fig. 1 for a sample section. In the form-finding step, the equilibrium shape in the absence of external loading is calculated, which is the shape at the beginning of an FSI simulation. Then in the FSI analysis, the interaction between the membrane and the fluid flow is simulated. The FSI analysis is followed by evaluating the design in terms of aerodynamic and structural characteristics of the blade. The cycle could be repeated for a new design to realize a better aerodynamic performance of the blade.

The goal of the current contribution is to make a comparison between a membrane blade and its conventional rigidblade counterpart. The NASA-Ames Phase VI rotor (Hand et al., 2001) is chosen as the reference rigid-blade configuration. The membrane blade uses the same planform as the NASA-Ames Phase VI rotor blade. Along the span, the blade is divided into four segments of equal span. The upper and lower surface of each segment is a prestressed membrane. The membranes are both connected to a prestressed cable at the trailing edge.

This paper has the following structure. Section 2 describes the membrane blade concept and the procedure of its design and analysis, followed by the theory of fluid and structure part as well as the coupling concept used in FSI simulations. Next, in Sect. 3, the model set-up for the simulations and the results are presented. The paper closes with Sect. 4, where the conclusions are summarized. 


\section{Design update}
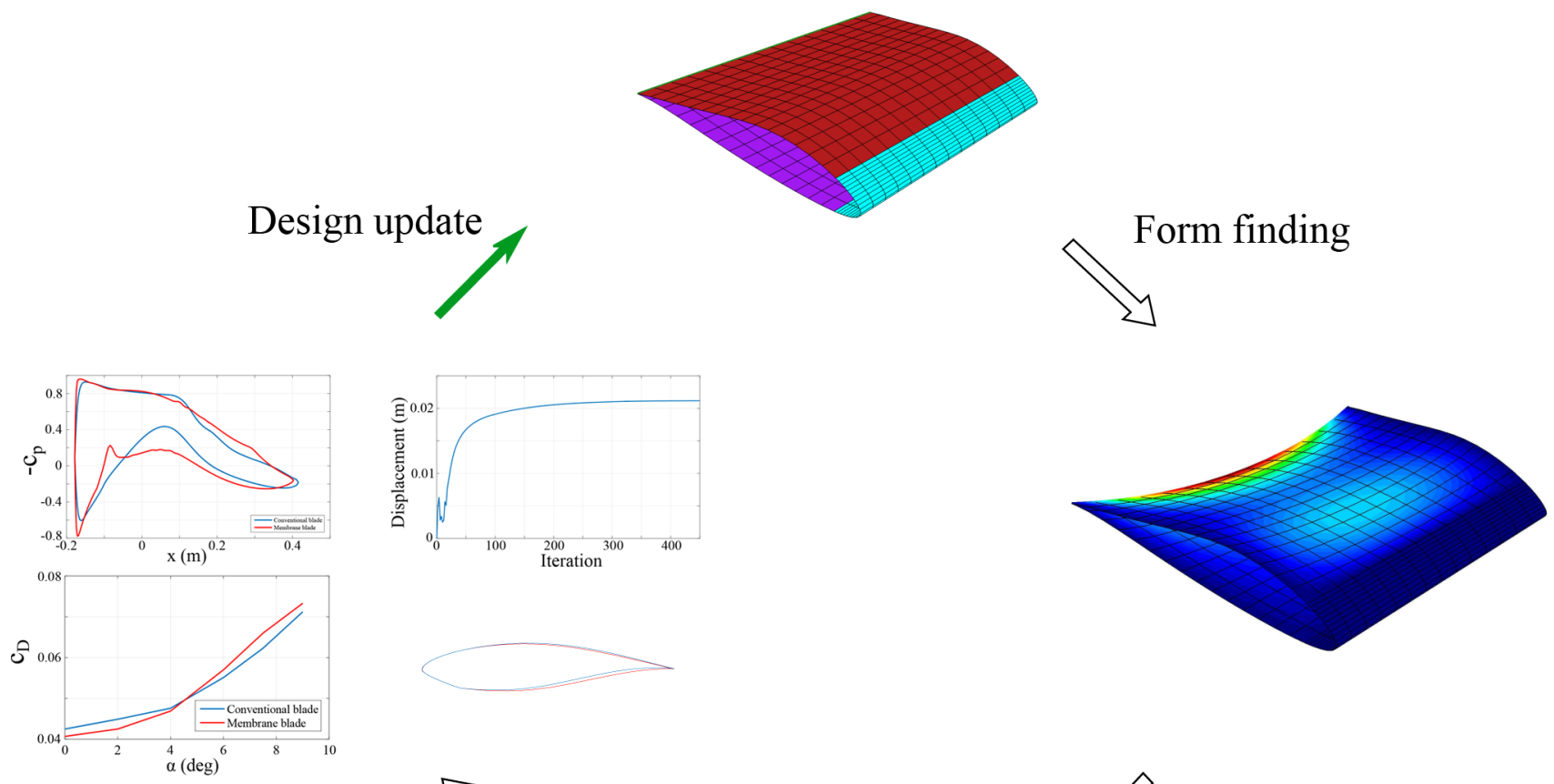

Design evaluation
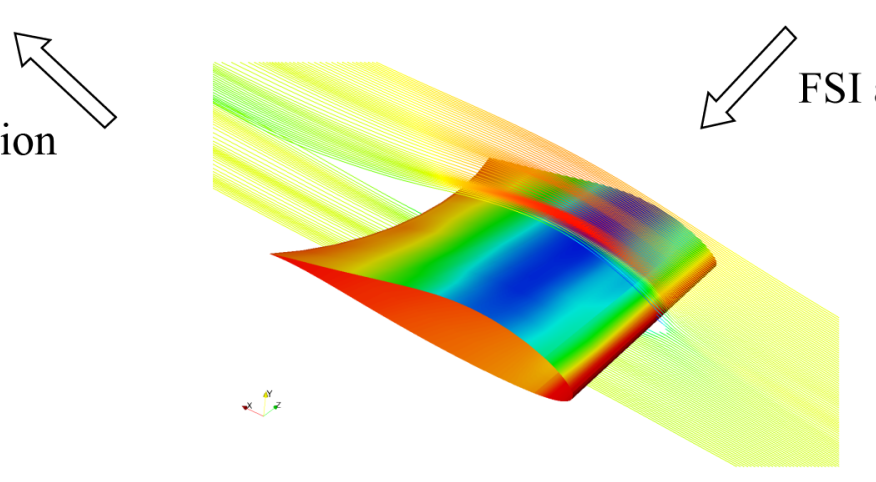

Figure 1. Analysis workflow for the membrane blade.

\section{Membrane blade concept and FSI simulation strategy}

Figure 2 shows the membrane wing concept studied in the current work. A rigid mast forms the leading edge section of the wing. To support the upper and lower membranes, ribs are mounted along the span of the wing and their number depends on the span length of the wing. Upper and lower membranes are joined together at the trailing edge via a pretensioned edge cable.

In the case of a flexible membrane blade, the shape of the blade's surface depends on the one hand on the working conditions in terms of wind speed, angle of attack, etc., and on the other hand on the structural properties of its supporting frame and the membranes which form the wing surface. The pressure distribution on the surface of the wing depends on the form and the above-mentioned working condition. The structural properties of the wing govern its deformation under such a loading. The final form of the wing results from this interaction between loading and displacement. This em-

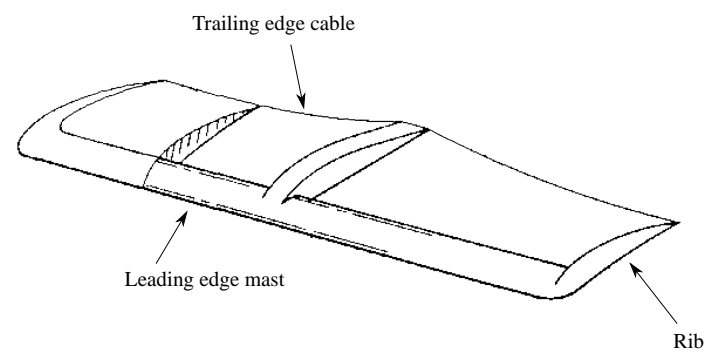

Figure 2. Sailwing construction concept from Ormiston (1971).

phasizes the necessity of FSI simulations for the analysis of such a wing concept. FSI simulations are computationally complex and time-consuming. From the fluid point of view, turbulent flow should be simulated using either large eddy simulation (LES) or Reynolds-averaged Navier-Stokes (RANS) turbulence models, and on the structural side nonlinear dynamic or static analysis is needed in order to correctly deal with large displacements which occur in the membranes. 
The analysis starts with a form-finding simulation. In form finding, the equilibrium state of the wing is calculated; i.e. the state in which membrane and edge cable internal forces are in equilibrium is computed. This is the initial shape of the blade surface in the absence of external forces. FSI simulations are started from this initial state. In the following sections, fluid, structure, and coupling-related aspects are explained in detail. The overall simulation work flow is shown in Fig 3, which demonstrates the sequence of the simulations.

The process of solving the flow problem for both approaches is presented in the figure as well. At each coupling iteration the fluid solver receives the displacement from the structural solver and updates the mesh. Then the fluid problem is solved for the updated mesh. It should be noted that in solving the problem using the finite-volume method, all the steps include operations performed on a three-dimensional mesh, while in the panel method the mesh consists of twodimensional surface discretization. In the case of the mesh update in particular, for the panel method discretization updating the mesh means adding the displacement at each node to the original coordinate of the node, but for the threedimensional volume mesh, in addition to applying the displacement on the boundary the displacement of the interior points should also be calculated. In addition to the higher computational cost of the three-dimensional mesh morphing, it is also a challenge to keep the quality of the volume elements as they deform.

\subsection{Fluid model}

Two approaches of very different fidelity are used for the modelling and simulation of fluid flow. The first one is the finite-volume-based numerical solution of Navier-Stokes equations using OpenFOAM and the second one is the vortex panel method. The advantage of the vortex panel method is that it is computationally less demanding, while its drawback is that it neglects the viscous effects. Still it is a very good alternative to Navier-Stokes during the early design stages. While a steady-state FSI simulation using the first approach takes about $10 \mathrm{~h}$ to converge, the same simulation takes about $20 \mathrm{~min}$ to converge to the steady-state solution using the vortex panel method (the same machine $(3.40 \mathrm{GHz}, 8 \mathrm{M}$ Cache, 15 GB RAM) was used for both approaches). Even though some details of fluid flow are neglected in the vortex panel method, the fact that it is much faster than solving NavierStokes equations enables design space explorations at reasonable computational costs in a certain range of operating conditions.

\subsubsection{Navier-Stokes equations}

The Navier-Stokes equations are the general equations describing the flow of fluid substances. For incompressible fluid flow with constant viscosity and density they read

$\nabla \cdot(\rho \boldsymbol{v})=0$ and

$$
\rho\left(\frac{\partial \boldsymbol{v}}{\partial t}+\boldsymbol{v} \cdot \nabla \boldsymbol{v}\right)=-\nabla p+\mu \nabla^{2} \boldsymbol{v}+\boldsymbol{f}
$$

Velocity $(\boldsymbol{v})$ and pressure $(p)$ fields are coupled in these equations. The SIMPLE algorithm of Patankar and Spalding (Patankar and Spalding, 1972) is used to enforce the coupling. A Reynolds-averaged Navier-Stokes model is used for turbulence modelling, and turbulent viscosity is modelled using a $k-\omega$ SST model (Menter, 1994). It is a two-equation model used to calculate the kinematic eddy viscosity. First the equations for turbulent kinetic energy $(k)$ and specific dissipation rate $(\omega)$ are solved. Kinematic eddy viscosity is then calculated from $k, \omega$, and other parameters of the model.

Near the no-slip boundaries, normal gradients become larger as the distance to the wall tends to 0 and viscous effects become more important. Usually the region near the wall is not directly resolved via the numerical model, but the so-called law of the wall, also known as wall function, is used to model the flow behaviour in this region. At the blade's surface, OpenFOAM wall functions are used: the kqRWallFunction for $k$ and omegaWallFunction for $\omega$. The wall functions set their corresponding parameter, $\omega$ or $k$, for the first node in the normal direction to the boundary. The boundary condition is set based on the logarithmic law if $y^{+}>11.5$ and the linear law if $y^{+}<11.5$.

Apart from solving the fluid flow problem, in an FSI simulation the fluid solver should take care of the displacement in the FSI interface and needs to update the mesh at each iteration, since the fluid domain changes after each iteration. As a result, the mesh needs to be updated correspondingly and it is important for the mesh update strategy that the quality of the initially good mesh should be preserved during the deformation. One solution could be re-meshing of the updated domain at each iteration, which is computationally very expensive. The other method is to stick with the initial mesh rather than generating a completely new mesh after each iteration and updating the initial mesh regarding the deformation of the FSI interface by solving the mesh deformation problem. Depending on the nature of the interface deformation, algebraic mesh motion solver or Laplacian-based solvers could be used. The algebraic method is used in cases where deformation of the mesh is governed by global motion laws like in rigid-body motion of bodies in the fluid domain and is less automatic compared with the Laplacian-based solver. In the Laplacian-based solvers, which are better candidates where the interface motion is less regular, the deformation at the FSI interface is used as a boundary condition for the mesh motion equation. The equation is then solved for calculating the displacement of the internal mesh nodes and their position is updated accordingly. We refer to Jasak (2009) and Jasak and Tukovic (2007) for more details on mesh motion solvers in OpenFOAM. 


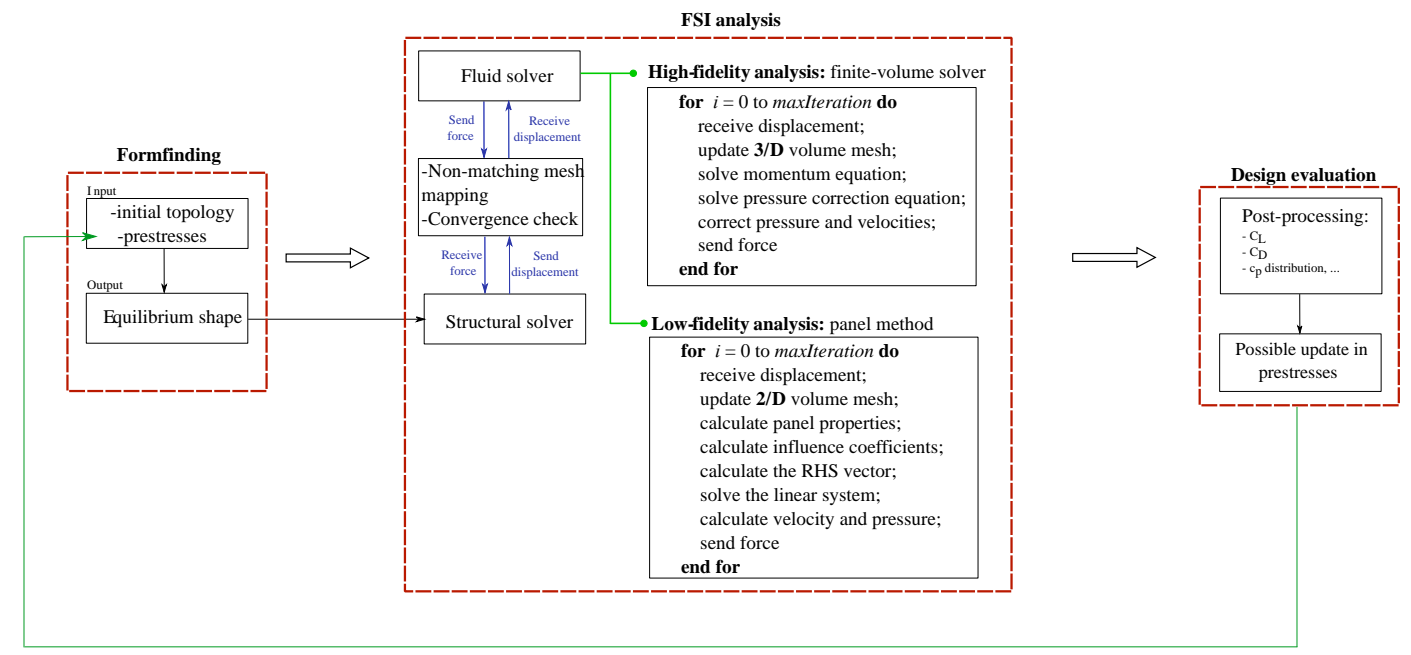

Figure 3. Schematic representation of the multi-fidelity analysis work flow.

\subsubsection{Vortex panel method}

The velocity field for the case of irrotational, incompressible, and inviscid flow can be represented by a velocity potential $\Phi$. This is the basis for the vortex panel method. The flow velocity can be calculated from the potential in the following way:

$u=-\frac{\partial \Phi}{\partial x}$,

$v=-\frac{\partial \Phi}{\partial y}$,

$w=-\frac{\partial \Phi}{\partial z}$.

Inserting the above equations into continuity Eq. (1) results in the continuity equation in terms of the potential:

$\nabla^{2} \Phi=0$

This equation can be solved by the superposition of elementary solutions. There are two boundary conditions for solving this Laplacian equation. One is that for a body submerged in fluid, the velocity component normal to the body's surface should vanish:

$\nabla \Phi \cdot \boldsymbol{n}=0$,

where $\boldsymbol{n}$ is the vector normal to the surface. The other condition is that the disturbance in the free-stream flow caused by the elementary solutions should vanish as the distance, $r$, from the boundary surface increases:

$\lim _{r \rightarrow \infty} \nabla \Phi=0$.

Using Green's identity, it can be shown that the potential at each point, $P$, inside the domain can be calculated in terms of the potential $(\Phi)$ and its derivative $\left(\frac{\partial \Phi}{\partial \boldsymbol{n}}\right)$ on the boundary of the domain:

$\Phi(P)=\frac{1}{4 \pi} \int_{S}\left(\frac{1}{r} \nabla \Phi-\Phi \nabla \frac{1}{r}\right) \cdot \boldsymbol{n} \mathrm{d} S$.

A detailed derivation of Eq. (9) is available in Katz and Plotkin (2008). The problem is now reduced to finding the values of the potential on the boundary which fulfill the continuity Eq. (6) and the two boundary conditions stated in Eqs. (7) and (8). The Laplace equation is a linear equation and if two functions fulfill the equation, their linear combination fulfills the equation as well. Hence, the solution of the continuity equation can be found as a superposition of elementary solutions like sources and doublets on the boundary of the domain.

In the panel method the strength of these singular solutions is calculated using the boundary condition stated in Eq. (7) to enforce zero normal velocity at the surface of the boundary. The surface of the wing is discretized with a number of panels as shown in Fig. 4. Each panel on the wing surface represents a quadrilateral source and a quadrilateral doublet element. In addition to wing panels there are wake panels to represent the wake behind the wing. Wake panels consist of quadrilateral doublets.

The Dirichlet implementation of the zero normal flow boundary condition is used for the collocation point at each panel. The collocation point of a panel is at the centre of the panel (in the case of the Dirichlet boundary condition the collocation points are infinitesimally shifted inside the body). The velocity at collocation point $P$ is calculated by summing up the contribution of each panel to the velocity at this point:

$\boldsymbol{v}(P)=\sum_{k=1}^{N} \mathbf{C}_{k} \mu_{k}+\sum_{l=1}^{N_{\mathrm{w}}} \mathbf{C}_{l} \mu_{l}+\sum_{k=1}^{N} \mathbf{B}_{k} \sigma_{k}$, 


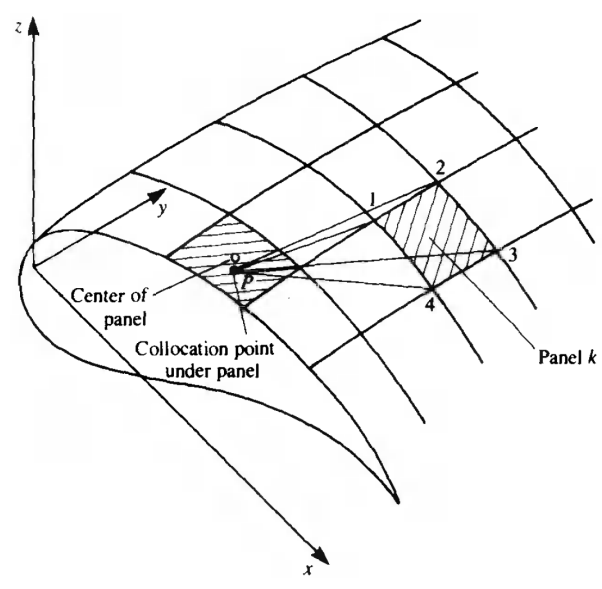

Figure 4. Discretization of the wing surface into panels from Katz and Plotkin (2008).

where $N$ is the number of panels on the wing's surface and $N_{\text {w }}$ is the number of wake panels. The first term in Eq. (10) is for the contribution of doublet elements on the wing panel, the second represents the contribution of doublet element at the wake, and finally the last one is for source terms on the wing. $\mathbf{C}_{k}$ can be interpreted as the velocity caused by the $k$ th panel at point $P$; it is calculated for a panel of unit strength. The same interpretation holds for $\mathbf{B}_{k}$ regarding the source terms. For more details on the calculation of the influence coefficients $\mathbf{C}_{k}$ and $\mathbf{B}_{k}$, we refer the reader to Katz and Plotkin (2008). The total velocity at point $P$ is the velocity caused by the panels plus the free-stream velocity. To set the total velocity in the normal direction to the panel to zero, the contribution of panels should cancel out that of the free-stream velocity:

$$
\left(\sum_{k=1}^{N} \mathbf{C}_{k} \mu_{k}+\sum_{l=1}^{N_{\mathrm{w}}} \mathbf{C}_{l} \mu_{l}+\sum_{k=1}^{N} \mathbf{B}_{k} \sigma_{k}\right) \cdot \boldsymbol{n}=-\boldsymbol{v}_{\infty} \cdot \boldsymbol{n} .
$$

Equation (11) should hold at every collocation point. Applying this equation to each collocation point, we end up with a system of $N$ equations with $N$ unknowns. This system of linear equations is then solved for the unknowns, which are the strengths of the doublet panels. It should be mentioned that the strength of the $k$ th source panels is already set to

$\sigma(k)=\boldsymbol{v}_{\infty} \cdot \boldsymbol{n}(k)$,

where $\boldsymbol{v}_{\infty}$ is the free-stream velocity and is moved to the right-hand side before solving the system of linear equations. In Eq. 12, $\boldsymbol{v}_{\infty}$ is the free-stream velocity vector. The strength of wake panels is also calculated in terms of doublet strength at the upper and lower neighbouring panels of the trailing edge (Fig. 5) using the Kutta condition.

The Kutta condition implies that the circulation at the trailing edge should be zero. Three panels intersect at the trailing edge. These are the wake panel and the two wing panels on

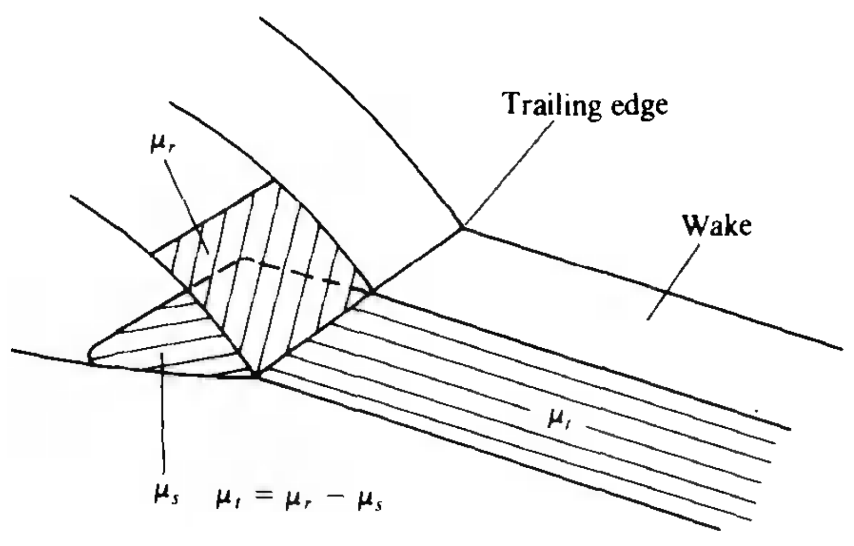

Figure 5. Wake panels used to apply the Kutta condition from Katz and Plotkin (2008).

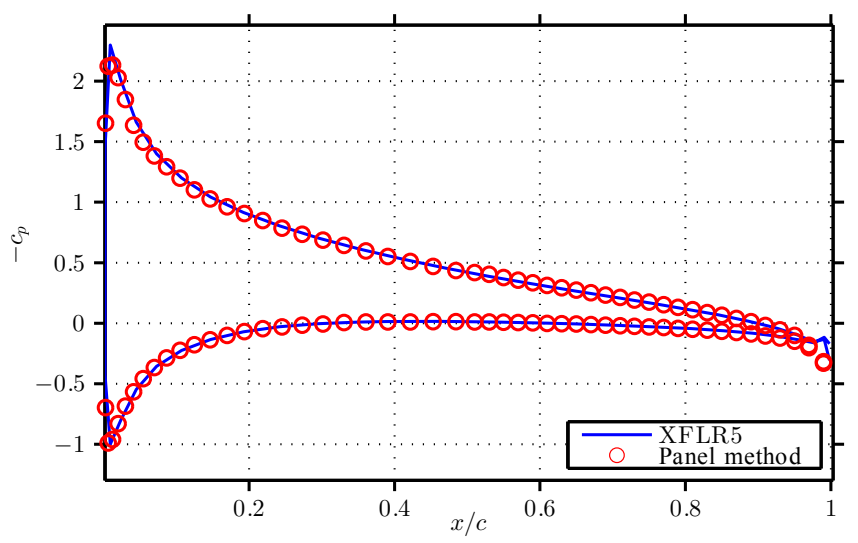

Figure 6. Pressure distribution over a NACA0012 wing section, $\alpha=6^{\circ}$.

the upper and lower surface of the wing. The Kutta condition is satisfied by setting the difference in the strength of the upper and lower panel to the wake strength:

$\mu_{\mathrm{w}}=\mu_{\text {upper }}-\mu_{\text {lower }}$.

Strength of the doublet panels are calculated by solving the resulting system of linear equations. In the post-processing step the velocity at the points of interest, which are the collocation points in particular, is calculated. The pressure is then calculated from the steady-state Bernoulli equation.

Figure 6 shows the calculated pressure distribution in the middle section of a NACA0012 wing for an angle of attack of $6^{\circ}$. The results are compared with the ones from XFLR5 to check the correctness of the developed panel solver which is used in the remainder of this publication as a low-fidelity fluid solver.

\subsection{Structural model}

The simulation of membrane structures typically consists of two steps: form-finding and nonlinear static or dynamic anal- 

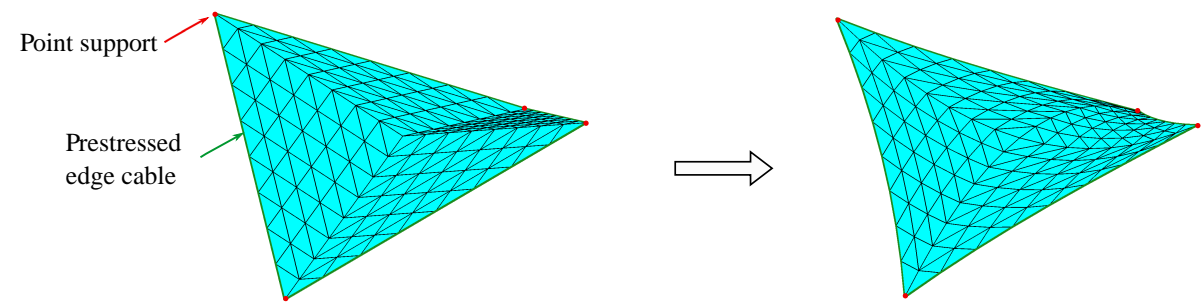

Figure 7. Form-finding analysis of a four-point tent. Left: initial state. Right: equilibrium state.

ysis. In general, structural analysis of membranes using the finite-element method is used and displacements are calculated for a specific structure under an applied load. Form finding of membrane structures can be seen as the inverse problem of structural analysis. Prestressed membrane structures can be supported at the edges by pretensioned edge cables. In the inverse problem of form-finding, the stresses in membrane and edge cables are given and support conditions (fixed boundaries) are defined. The goal of the form-finding analysis is to find the shape at which an equilibrium between structural forces exists. In other words, form-finding analysis calculates the equilibrium shape of the membrane enclosed by a given boundary and with predefined stress distributions. It has been inspired, e.g., by the works of the German architect, Frei Otto (Otto and Rasch, 1995) and was originally developed for form finding of cable structures. Form finding could be done using different approaches like the force density method (Schek, 1974), dynamic relaxation (Wakefield, 1999), or the updated reference strategy (URS) (Bletzinger and Ramm, 1997; Wüchner and Bletzinger, 2005). We have used the URS-based method available in the in-house structural solver CARAT++. As a classical form-finding example, the four-point tent is presented in Fig. 7. The reference configuration of the four-point tent example consists of four flat membrane patches. They are connected to four edge cables at the boundaries and are fixed at four support points. After applying prestresses to the four membranes and the supporting edge cables, the structure evolves from flat membranes to a double curved surface where internal membrane prestresses are in equilibrium with the forces from the four edge cables.

CARAT++ has also been used for performing static nonlinear finite-element method (FEM) analysis using the loadcontrol method. This starts from the equilibrium state and computes the deformations due to the external loads caused by the fluid flow.

\subsection{Fluid-structure interaction}

Fluid-structure interaction studies the interactions between a solid body and its surrounding fluid. The interface between the fluid field $\left(\Omega_{\mathrm{F}}\right)$ and the structure field $\left(\Omega_{\mathrm{S}}\right)$ is designated by $\Gamma_{\mathrm{I}}$. The load from the fluid field is coupled with the displacement from the structure field. There are two coupling conditions enforced at the interface:

\subsubsection{Kinematic continuity condition}

Enforcing this constraint ensures that the fluid and structure interfaces lie on each other during the simulation. It is satisfied if the displacement at the fluid interface is the same as the displacement at the solid interface:

$\boldsymbol{d}_{\Gamma_{\mathrm{I}}}^{\mathrm{F}}(t)=\boldsymbol{d}_{\Gamma_{\mathrm{I}}}^{\mathrm{S}}(t)$

\subsubsection{Dynamic continuity condition}

Dynamic continuity condition is about mapping the correct force vector from the fluid interface to the solid interface. It implies

$f_{\Gamma_{\mathrm{I}}}^{\mathrm{F}}(t)=f_{\Gamma_{\mathrm{I}}}^{\mathrm{S}}(t)$.

The interface mesh at the fluid side is in most cases finer than the mesh at the structure side. Non-matching mesh mapping techniques are necessary to map the equivalent nodal force and nodal displacement at the FSI interface. A mortar mapping method is used in the current work. A basic criterion for mapping algorithms is consistency. It implies that a constant field is mapped exactly from one mesh to the other mesh. Another criterion is the conservation of energy, which is used to derive the so-called conservative mapping operators. In conservative mapping, total energy is conserved as the fields are mapped between the meshes at the interface. The conservation of interface energy reads

$$
\int_{\Gamma} \boldsymbol{d}_{\Gamma}^{\mathrm{F}^{T}} \boldsymbol{f}_{\Gamma}^{\mathrm{F}} \boldsymbol{d} \Gamma=\int_{\Gamma} \boldsymbol{d}_{\Gamma}^{\mathrm{S} T} \boldsymbol{f}_{\Gamma}^{\mathrm{S}} \boldsymbol{d}_{\Gamma} .
$$

Normal and dual mortar algorithms for mapping are not consistent in general. A novel technique for enforcing consistency on the mapping algorithm by scaling up the structural shape functions for the calculation of mapping matrices is utilized. For the details of the formulation and its implementation, we refer to Wang et al. (2016).

There are two classes of methods for tackling an FSI problem: monolithic and partitioned solution schemes. In the monolithic approach, fluid and structure equations are merged into a single system of equations and are solved simultaneously, while in the partitioned approach the problem 


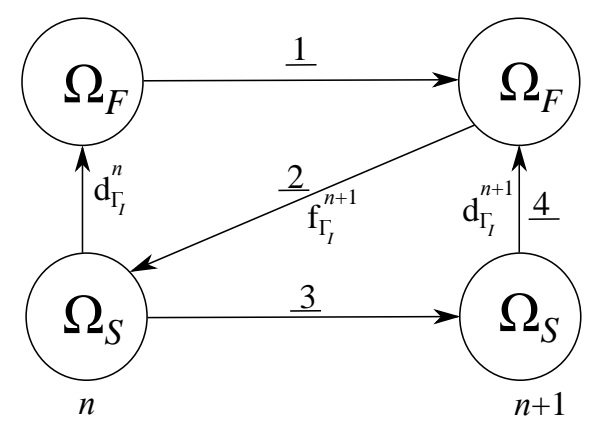

Figure 8. Solution procedure for the coupled problem.

is divided into two separate sub-problems for fluid and structure field (Wüchner et al., 2007). Each field is solved separately in the partitioned approach and the exchange of information takes place in a separate coupling step. The monolithic approach typically has an advantage over the partitioned one in terms of stability and accuracy (Kupzok, 2009). On the other hand, solving the two fields independently in a modular environment provides the possibility of using the most efficient available solution techniques for each field. Thus, this is the preferred simulation approach to develop the multi-fidelity analysis concept.

For a steady-state FSI simulation, the coupling steps in each iteration are as follows:

1. Solve the fluid problem for the new iteration $(n+1)$.

2. Send the resulting force at the interface to the structure solver.

3. Solve the structure problem.

4. Send the calculated displacement to the fluid solver and proceed to the next iteration.

Schematic representation of these steps is shown in Fig. 8.

In the following the structure problem is abbreviated by the operator $S$ and the fluid problem by the operator $F$. On the structure side the solver receives the loading from the fluid solvers and calculates the displacement at the interface:

$\boldsymbol{d}_{\Gamma_{\mathrm{I}}}^{n+1}=S\left(\boldsymbol{f}_{\Gamma_{\mathrm{I}}}^{n+1}\right)$.

For the fluid solver it is just the opposite; it receives the displacement at the interface and delivers the load applied by the fluid:

$f_{\Gamma_{\mathrm{I}}}^{n+1}=F\left(d_{\Gamma_{\mathrm{I}}}^{n}\right)$.

Inserting $\boldsymbol{d}_{\Gamma}$ from Eq. (17) into Eq. (18), we have

$\boldsymbol{d}_{\Gamma_{\mathrm{I}}}^{n+1}=S\left(\boldsymbol{f}_{\Gamma_{\mathrm{I}}}^{n+1}\right)=S\left(F\left(\boldsymbol{d}_{\Gamma_{\mathrm{I}}}^{n+1}\right)\right)=S^{\circ} F\left(\boldsymbol{d}_{\Gamma_{\mathrm{I}}}^{n}\right)$.

Equation (19) could be solved either using fixed-point iteration-based methods or Newton-based methods. In the

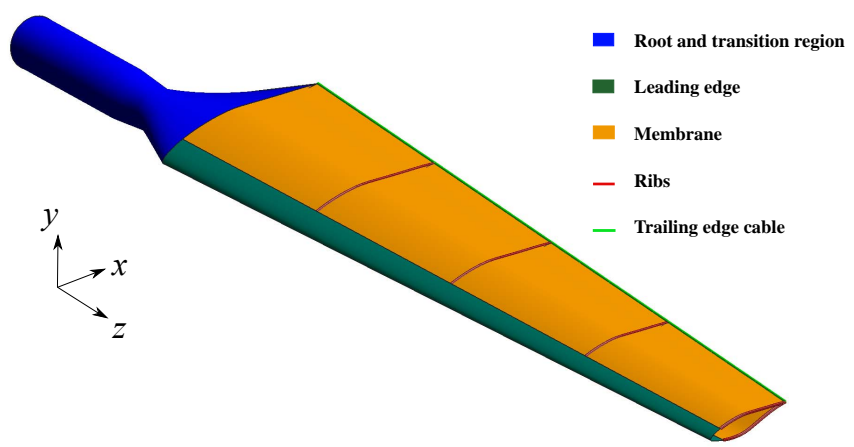

Figure 9. Blade planform.

current work, the Gauss-Seidel method is used to solve the equation iteratively. The convergence of the iterative solution procedure is checked during the iteration steps by comparing the current solution with the previous solution. A relative tolerance of $10^{-6}$ is used as the convergence criterion.

Table 1. Membrane properties (u: upper; 1: lower).

\begin{tabular}{ll}
\hline$E$ & $84 \mathrm{MPa}^{-3}$ \\
$\rho$ & $1400 \mathrm{~kg} \mathrm{~m}^{-3}$ \\
$t$ & $0.48 \mathrm{~mm}$ \\
$\sigma_{\text {chordwise }}^{\mathrm{u}}$ & $180 \mathrm{kPa}$ \\
$\sigma_{\text {spanwise }}^{\mathrm{u}}$ & $480 \mathrm{kPa}$ \\
$\sigma_{\text {chordwise }}^{1}$ & $180 \mathrm{kPa}$ \\
$\sigma_{\text {spanwise }}^{1}$ & $480 \mathrm{kPa}$ \\
\hline
\end{tabular}

Table 2. Trailing edge cable properties.

\begin{tabular}{ll}
\hline$E$ & $125 \mathrm{GPa}^{-3}$ \\
$\rho$ & $7800 \mathrm{~kg} \mathrm{~m}^{-3}$ \\
radius & $4 \mathrm{~mm}$ \\
$\sigma$ & $30 \mathrm{MPa}$ \\
\hline
\end{tabular}

Table 3. Rib properties.

\begin{tabular}{ll}
\hline$E$ & $190 \mathrm{GPa}$ \\
$\rho$ & $7800 \mathrm{~kg} \mathrm{~m}^{-3}$ \\
$A$ & $2 \mathrm{~cm} \times 12 \mathrm{~cm}$ \\
\hline
\end{tabular}

\section{Model set-up and results}

The studied membrane blade can be seen in Fig. 9. It is inspired by the layout of the rigid NASA-Ames Phase VI rotor with a length of $5.029 \mathrm{~m}$ (Hand et al., 2001). The chord length varies along the blade, from $0.73 \mathrm{~m}$ at the root of the blade (after the transition from cylindrical hub profile to the 


\section{Top view}
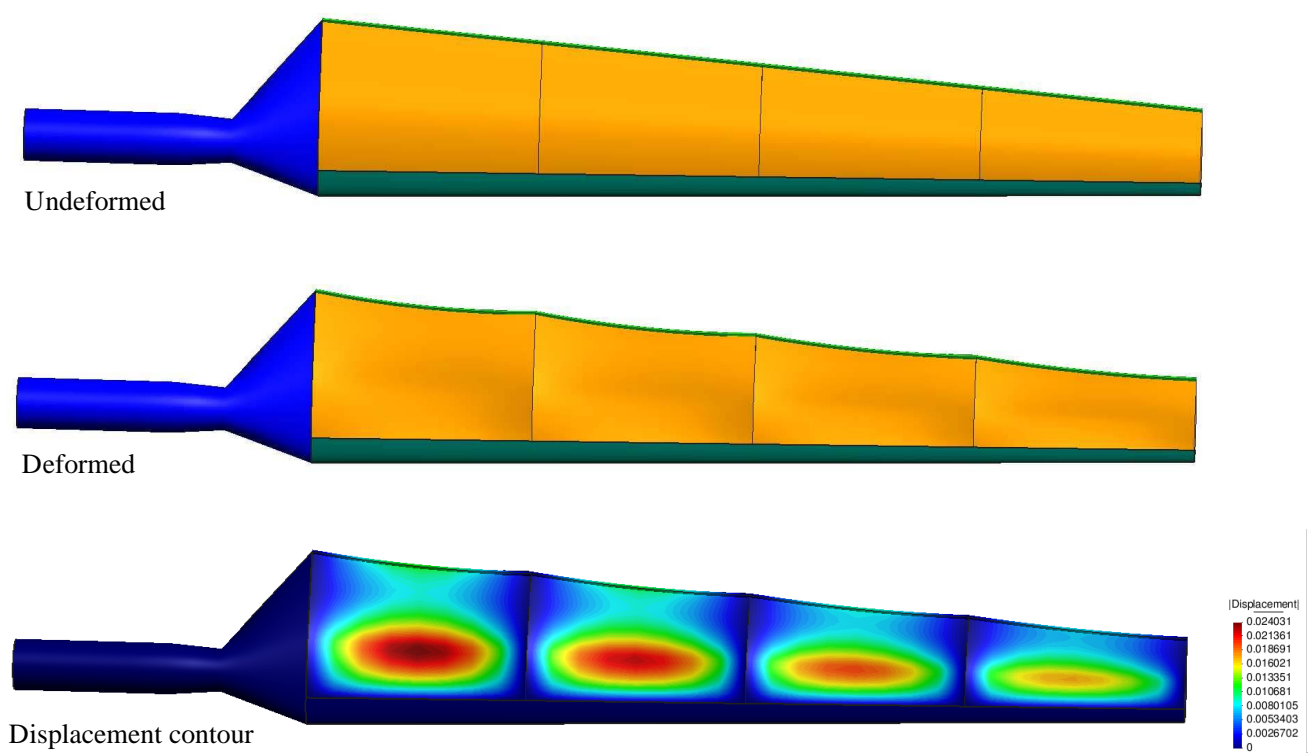

\section{Section view}

Undeformed

Deformed

Figure 10. Form finding of the membrane blade. Undeformed and deformed geometry from top and front views.

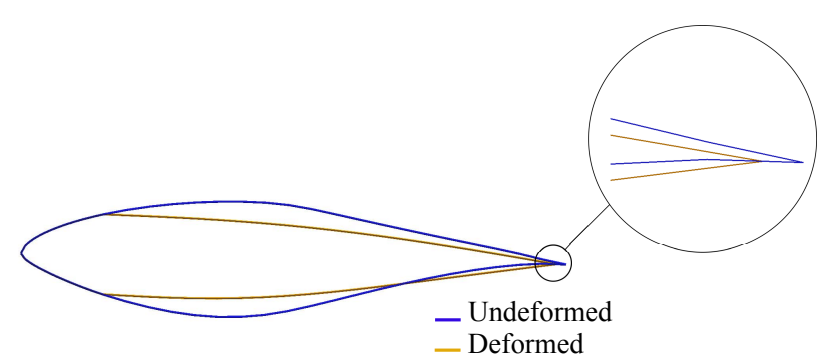

Figure 11. Form finding of the membrane blade (mid-span section, second segment from the root).

airfoil profile) to $0.35 \mathrm{~m}$ at the tip of the blade. Upper and lower membranes are wrapped around the rigid leading edge, which extends up to $15 \%$ of the chord length. The membranes are supported by four ribs and by an edge cable at the trailing edge. The four ribs divide the blade into four segments with equal span. The structural properties of the membranes, ribs (which are modelled as beams), and edge cable are summarized in Tables 1-3. The prestresses in Table 1 are for the first blade section from the root. The prestress in span

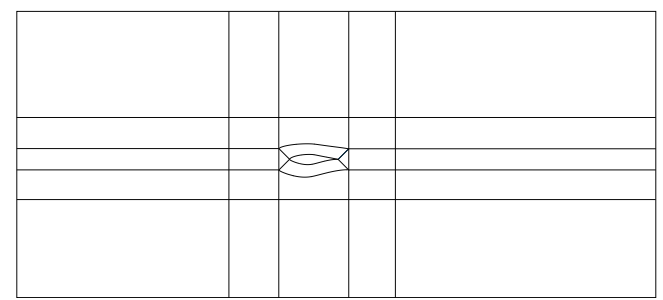

Figure 12. Blocking structure for the fluid mesh.

direction is the same for all four segments, since they all have the same span, but the prestress in chord direction is scaled with the mean chord length for the three other segments.

\subsection{Form finding}

The equilibrium state of the blade with structural parameters presented in the Tables 1-3 is calculated via form-finding analysis. The deformed state is compared with the undeformed state in Fig. 10. Membranes and the edge cables pull against each other, and as a result, the edge cables are moved 


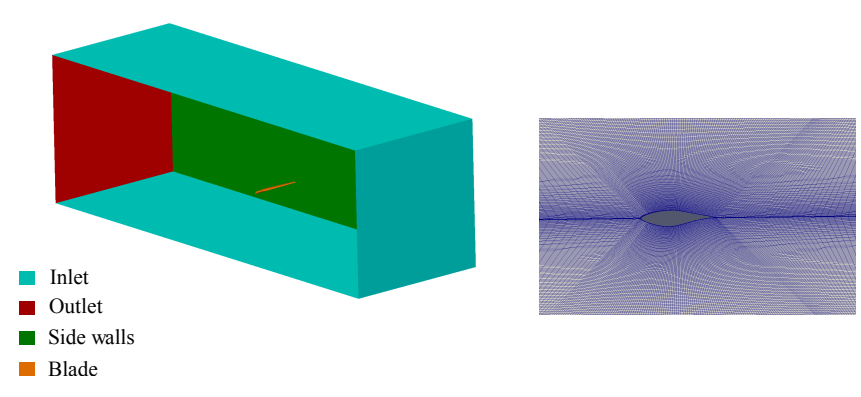

Figure 13. Left: fluid domain. Right: fluid mesh in the vicinity of the blade.
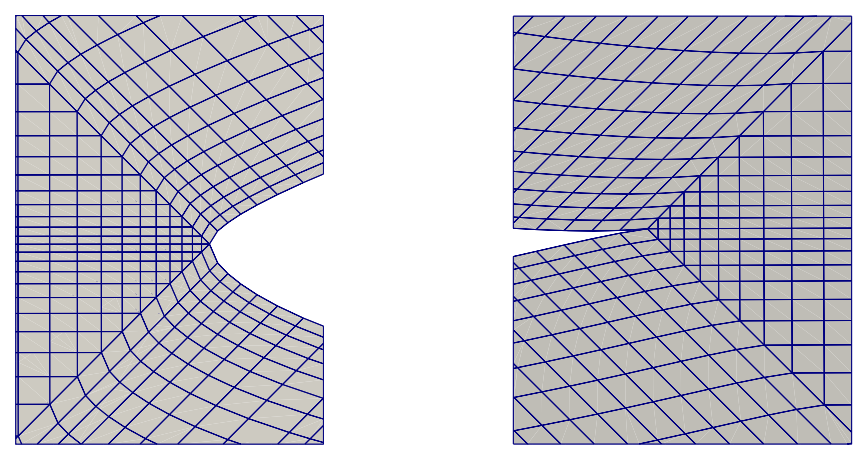

Figure 14. Detailed view of the fluid mesh at the leading edge and the trailing edge.

toward the leading edge with maximum displacement at the middle of each blade segment. The prestresses in the membranes form double-curved membrane surfaces, where the upper membranes are moved downwards and lower membranes are moved upwards. While the cross section remains unchanged at the four ribs, due to the deformation of the two membranes, the cross section of the blade changes continuously on other sections along the span. Figure 11 shows how the cross section at the middle of the second segment from the root deviates from the initial cross section (which is the S809 airfoil profile) and how the upper and lower membrane pull the elastic trailing edge cable toward the leading edge.

\subsection{Fluid set-up}

For the fluid side, simpleFoam solver from OpenFOAM has been used for performing steady-state computational fluid dynamics (CFD) simulations. The schematic representation of the blocking strategy is presented in Fig. 12. The computational domain together with the mesh in the vicinity of the blade is shown in Fig. 13. For a better presentation of the used mesh, detailed view of the elements structure at the leading edge and the trailing edge is shown in Fig. 14.

The domain size is $15 \mathrm{~m} \times 15 \mathrm{~m} \times 45 \mathrm{~m}$, which results in a blockage ratio of about $0.3 \%$. The tip of the blade has a distance of $10 \mathrm{~m}$ to the far-field boundary. The domain is discretized with a total of 2.9 million cells (hexahedral and

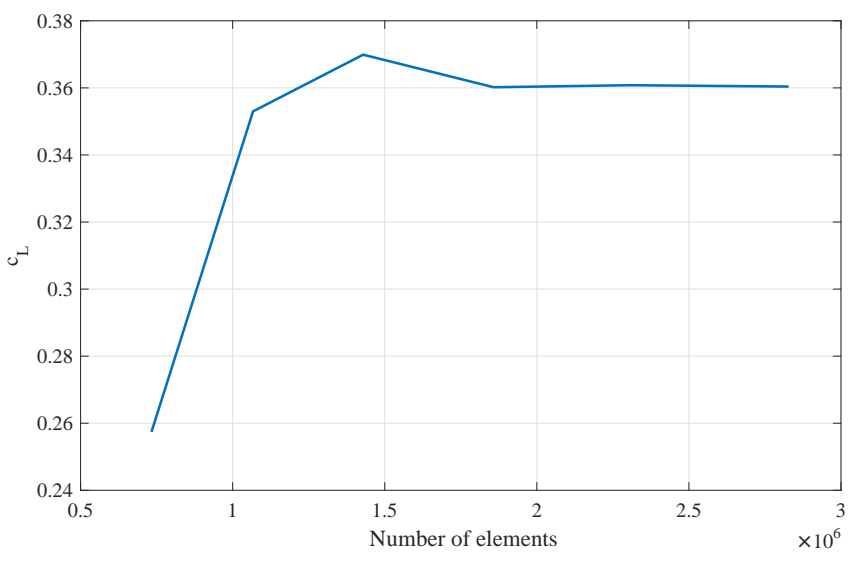

Figure 15. Mesh convergence study for the rigid blade, $\alpha=4.0^{\circ}$.

tetrahedral elements), which results in a maximum $y^{+}$value of about 70. Figure 15 presents the result of the mesh convergence study performed for the rigid-blade configuration at $\alpha=4.0^{\circ}$. As can be seen, $c_{\mathrm{L}}$ has converged for the mesh with 2.9 million elements.

The $k-\omega S S T$ model has been used. OpenFOAM wall functions are used at the blade's surface: kqRWallFunction for $k$ and omegaWallFunction for $\omega$. The velocity at the inlet is $30 \mathrm{~m} \mathrm{~s}^{-1}$. The boundary conditions for fluid simulation are summarized in Table 4.

\subsection{FSI simulations}

FSI simulations were done for six different angles of attack from 0 to $9^{\circ}$. In the following, FSI_CFD is used for simulations using the finite-volume method on the fluid side and FSI_Panel is used for simulations which use the panel method for flow modelling. Because of blade's deformation in FSI simulations, the fluid solver should, in addition to solving the fluid flow problem, take care of the movement in the mesh as well. For the FSI_CFD case the deformation of the blade, which is applied to the blade patch, is diffused into the fluid domain. This means that the boundary motion is distributed into the volume mesh and the zero displacement condition is applied at the far-field boundaries of the fluid domain. To solve the mesh motion problem the displacement Laplacian-based solver from OpenFOAM is used with the quadratic inverse distance diffusion method.

Convergence to the steady-state solution when using the panel method solver is about 30 times faster than using the simpleFoam solver from OpenFOAM as the fluid solver. The panel code was run on a single processor, while for OpenFOAM simulations, 10 processors were used. For both cases a relaxation factor of 0.15 was used for the displacement field for all angles of attack except for $\alpha=7.5^{\circ}$ and $\alpha=9.0^{\circ}$, where the relaxation factor was reduced to 0.1 to improve stability in the FSI run. The relaxation factor $\left(\omega_{\mathrm{r}}\right)$ is applied in order not to send the total calculated displacement to the 
Table 4. Boundary conditions for computational fluid dynamics (CFD) simulations.

\begin{tabular}{lllll}
\hline Boundary & $U$ & $p$ & $k$ & $\omega$ \\
\hline Inlet & $\begin{array}{l}\text { Fixed value } \\
\text { uniform }\left(u_{\infty}, v_{\infty}, \omega_{\infty}\right)\end{array}$ & Zero gradient & $\begin{array}{l}\text { Fixed value } \\
\text { uniform } K=\frac{0.1 U_{\infty}^{2}}{R e_{L}}\end{array}$ & $\begin{array}{l}\text { Fixed value } \\
\text { uniform } \omega=\frac{10 \cdot O_{\infty}}{L}\end{array}$ \\
\hline Outlet & Zero gradient & $\begin{array}{l}\text { Fixed value } \\
\text { uniform } 0\end{array}$ & Zero gradient & Zero gradient \\
\hline Side walls & Symmetry plane & Symmetry plane & Symmetry plane & Symmetry plane \\
\hline Blade & $\begin{array}{l}\text { Fixed value } \\
\text { uniform }(0,0,0)\end{array}$ & Zero gradient & $\begin{array}{l}\text { kqRWallFunction } \\
\text { uniform } 1-10\end{array}$ & $\begin{array}{l}\text { Omega wall function } \\
\text { uniform } \omega=10 \frac{6 v}{\beta_{1}\left(\Delta d_{1}\right)^{2}}\end{array}$ \\
\hline
\end{tabular}
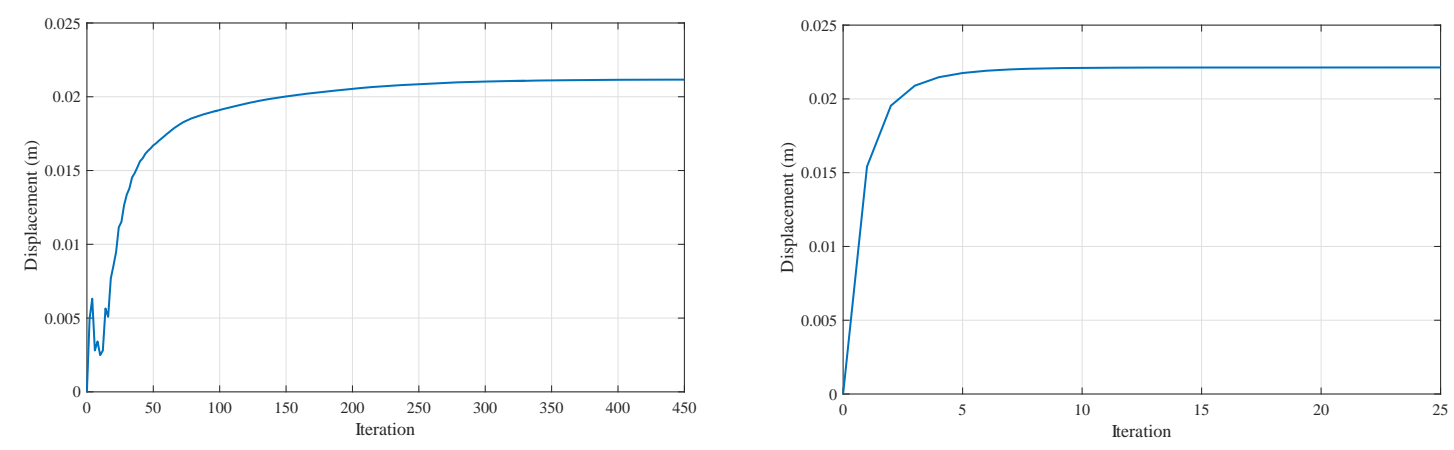

Figure 16. Convergence of the displacement for the selected monitor point. Left: FSI_CFD. Right: FSI_Panel.

Table 5. Comparison of displacement $(\mathrm{m})$ in $y$ direction for different angles of attack.

\begin{tabular}{llll}
\hline$\alpha(\mathrm{deg})$ & FSI_Panel & FSI_CFD & $\%$ diff \\
\hline 0.0 & 0.0175 & 0.0170 & 2.87 \\
2.0 & 0.0198 & 0.0189 & 4.74 \\
4.0 & 0.0221 & 0.0211 & 4.73 \\
6.0 & 0.0241 & 0.0231 & 4.38 \\
7.5 & 0.0252 & 0.0241 & 4.53 \\
9.0 & 0.0255 & 0.0237 & 7.68 \\
\hline
\end{tabular}

fluid solver in increments but to send a fraction of that to improve the stability of the coupling algorithm and preserve the quality of the mesh on the fluid side:

$\boldsymbol{d}_{\Gamma_{\mathrm{I}, \text { sent }}}^{n+1}=\omega_{\mathrm{r}} \boldsymbol{d}_{\Gamma_{\mathrm{I}, \text { calculated }}}^{n+1}+\left(1-\omega_{\mathrm{r}}\right) \boldsymbol{d}_{\Gamma_{\mathrm{I}}}^{n}$.

The relaxation factor should be kept below a certain limit (which is case dependent) for FSI_CFD simulations, otherwise the quality of the finite-volume mesh cannot be preserved during the simulation and the simulation might crash as a result of having highly distorted elements in the mesh. The same relaxation factor is used for the FSI_Panel case. The reason for using the same relaxation factor is to have a fair comparison between the convergence behaviour of the two approaches, but it must be mentioned that for FSI_Panel cases a higher relaxation factor can be used as well to have faster convergence without getting stability problems due to distorted element in the mesh, which in this case is just a discretized surface.

First, we compare the convergence behaviour of the displacements to the steady-state solution for each approach (Fig. 16). Displacement of the point in the mid-span section of the second segment from the root with $x / c=0.5$ is compared ( $c$ is the chord length). While with the panel method convergence is reached within 25 iterations, FSI simulation using simpleFoam takes 450 iterations to converge. The time each iteration takes is also different in the two approaches. Overall, in the case of the panel method, convergence is reached approximately 30 times faster. For this particular case, the FSI_CFD simulation has taken about $10 \mathrm{~h}$ to converge using 10 processors, but FSI_Panel has converged within about $20 \mathrm{~min}$ on a single processor. For $\alpha=4^{\circ}$ the converged displacement is $0.0221 \mathrm{~m}$ from FSI_Panel and $0.0211 \mathrm{~m}$ from FSI_CFD, which demonstrates clearly the applicability of the low-fidelity approach, provided that the blade-operating condition is in agreement with the respective modelling assumptions (see Sect. 2.1.2).

The comparison of the two approaches for the selected monitor point is summarized in Table 5. For $\alpha=0^{\circ}$ the difference in the calculated displacement from the two approaches is $2.87 \%$. The difference increases with the angle of attack. For the base airfoil of the studied blade, the S809 

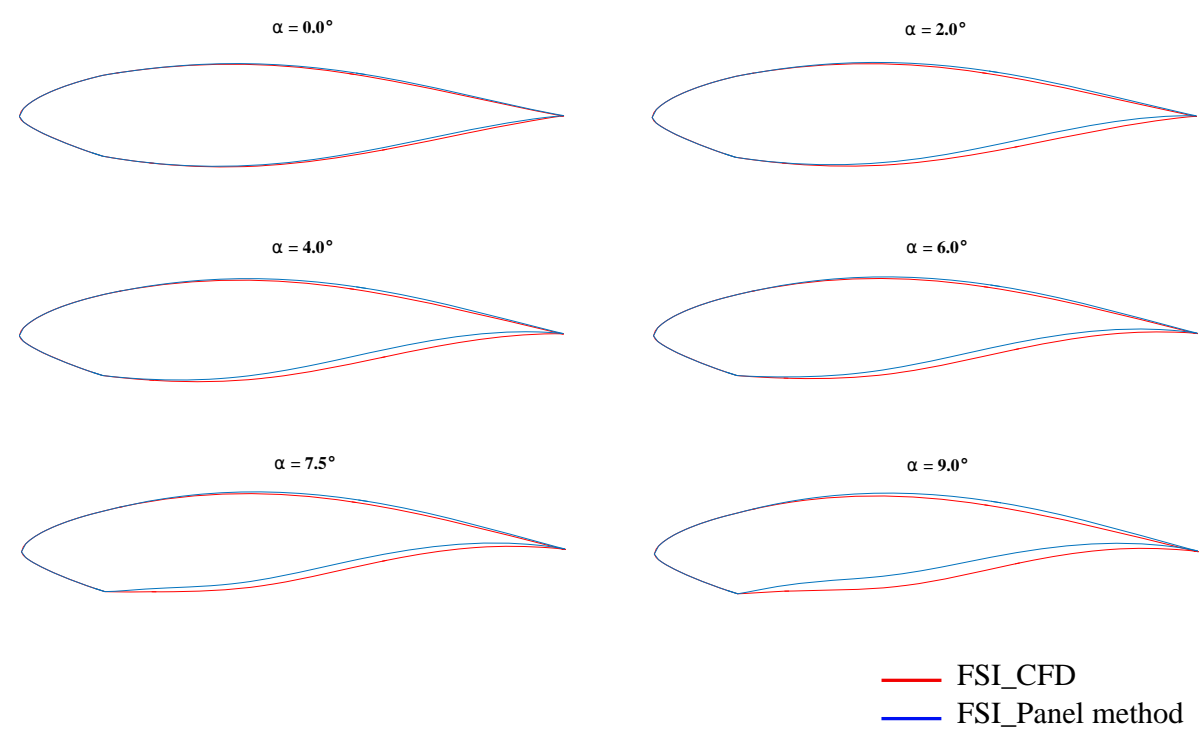

Figure 17. Comparison of the converged cross section in the mid-span section of the second segment from the root.
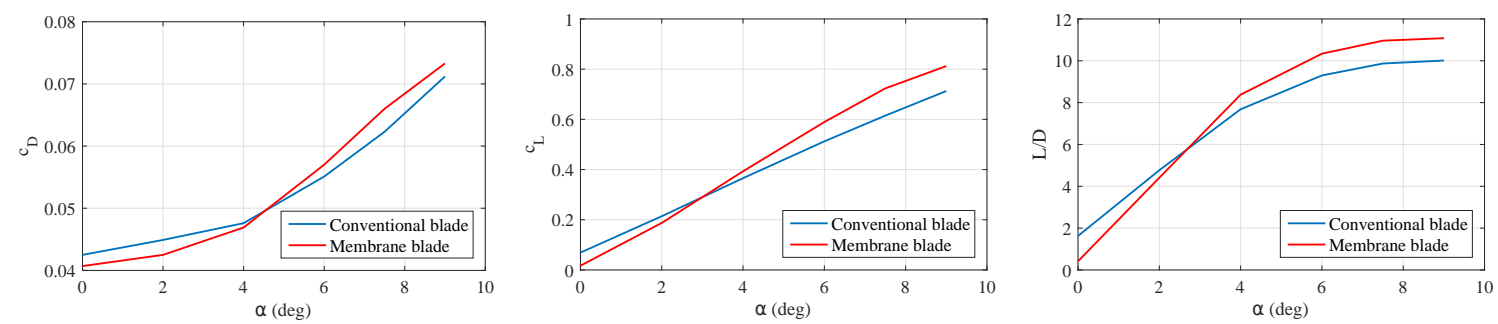

Figure 18. Comparison between the aerodynamic characteristics of the membrane blade and rigid blade.

profile, stall happens at $\alpha \approx 9^{\circ}$. With the emergence of stall and flow separation, the assumptions of the panel method are no more valid. This explains the increased deviation of the FSI_Panel result from the FSI_CFD result for $\alpha=9^{\circ}$.

After local comparison of the calculated displacements for the two approaches in Table 5 for a single monitor point, a more global comparison is made by comparing the converged cross section shape in the steady state. Figure 17 shows the cross section of the blade in the middle of the second segment from the root. For the upper surface of the blade, there is a good agreement between the two methods, even though the difference increases with the angle of attack, which is to be expected. The difference in the converged shape is higher for the lower surface and especially for $\alpha=7.5^{\circ}$ and $\alpha=9.0^{\circ}$. It can be explained by the increased discontinuity in the slope of the surface at the point at which the lower membrane is attached to the leading edge mast. At the point of attachment there is a kink, which is much more visible for higher angles of attack. The local flow separation downstream of the kink is not captured by the panel method, which results in different pressure distributions and as a consequence different converged shapes for the two approaches.

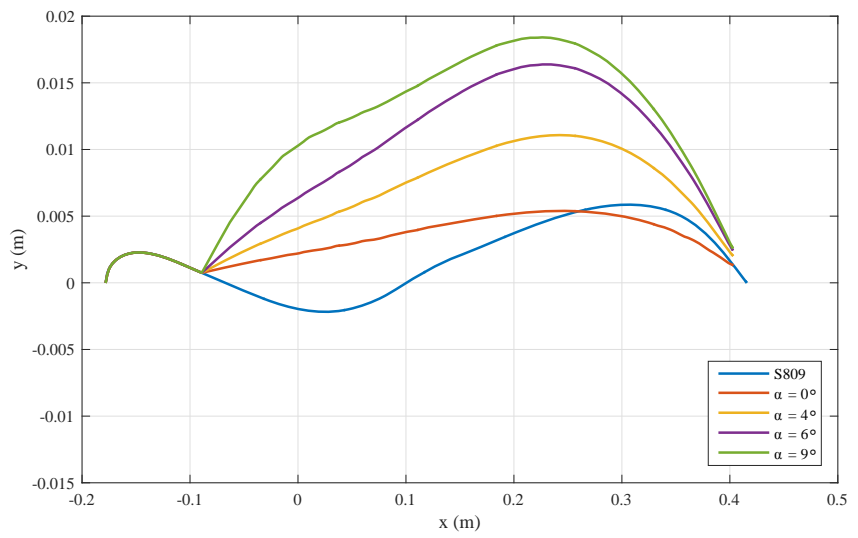

Figure 19. Comparison of the camber line in the middle span section of the second segment from the root.

The concept of a membrane blade facilitates a lighter blade construction due to the optimal load-carrying behaviour and also due to its capability to alleviate peak loadings by deformation. Its flexibility is also an advantage in terms of dynamic (e.g. due to gusts) loading applied to the blade. It should also have an improved performance compared to 
Table 6. Percentage change in aerodynamic characteristics of the membrane blade compared with the rigid blade.

\begin{tabular}{lrrrrrr}
\hline$\alpha(\mathrm{deg})$ & 0.0 & 2.0 & 4.0 & 6.0 & 7.5 & 9.0 \\
\hline$\Delta c_{L}$ & -75.5427 & -12.4708 & 7.4925 & 15.0273 & 17.6614 & 13.9290 \\
$\Delta c_{D}$ & -4.2353 & -5.3452 & -1.4706 & 3.4483 & 5.9390 & 2.9494 \\
$\Delta(L / D)$ & -74.4610 & -7.5280 & 9.0968 & 11.1931 & 11.0652 & 10.6650 \\
\hline
\end{tabular}

Table 7. Maximum camber and its location for the middle span section of the second segment from the root.

\begin{tabular}{lrrrrrr}
\hline$\alpha(\mathrm{deg})$ & 0.0 & 2.0 & 4.0 & 6.0 & 7.5 & 9.0 \\
\hline Maximum camber \% & 0.34 & 0.93 & 1.91 & 2.82 & 3.29 & 3.17 \\
Location of maximum camber & 71.48 & 73.53 & 73.51 & 71.45 & 69.40 & 69.40 \\
\hline
\end{tabular}

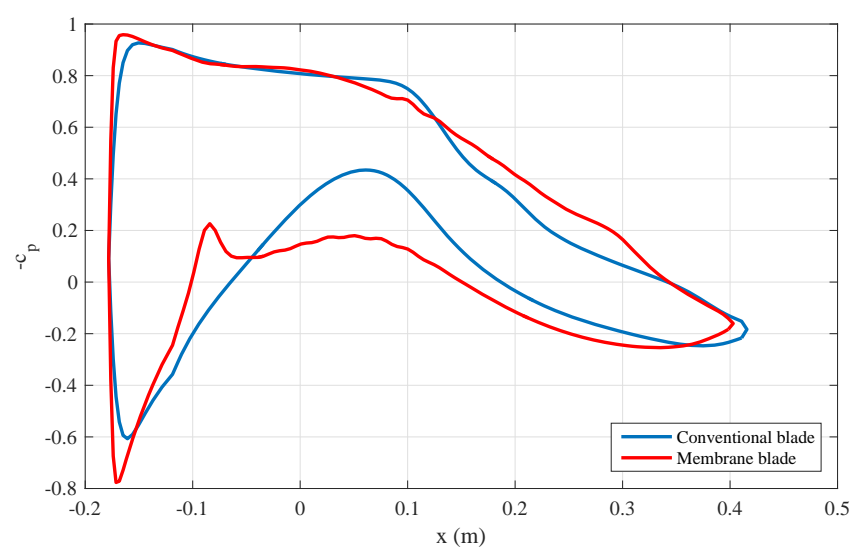

Figure 20. Pressure coefficient distribution over the middle span section of the second segment from the root.

rigid-blade configurations in stall region because of the socalled "soft stall characteristics" of the membrane wings (Maughmer, 1979). In order to assess the aerodynamic performance of the studied blade, the lift coefficient, drag coefficient, and lift-to-drag ratio of the blade are compared with the rigid-blade configuration (i.e. the configuration before form finding). As can be seen in Fig. 18, for smaller angles of attack the membrane blade has smaller lift and drag coefficient compared with the rigid blade; however, with the increase in the angle of attack, higher lift and drag coefficients are observed for the membrane blade. Table 6 provides a numeric comparison of the change in these coefficients compared with the rigid blade. The cross section of the membrane blade in the absence of an aerodynamic load is shown in Fig. 11 (the orange curve). The membrane blade has a pretty much symmetric profile in the unloaded state. For $\alpha=0.0^{\circ}$, the converged cross section is also a rather symmetric profile (Fig. 17). This explains the big decrease in the lift coefficient of the membrane blade at $\alpha=0.0^{\circ}$, compared with the rigid blade with the asymmetric S809 airfoil. With the increase in the angle of attack, the loading on the blade, and as a consequence the converged cross section profile, becomes more and more asymmetric, which can be seen in Fig. 17. Moreover the displacements in the membranes increase with the increase in the angle of attack. It increases the thickness of the blade profile and as a consequence there is an improvement in the lift coefficient of the membrane blade compared with the rigid blade for higher angles of attack. The drag coefficient increases as well with the increase in the angle of attack and the drag coefficient of the membrane blade is higher than that of the rigid blade. But the improvement in the lift coefficient is larger compared with the increase in the drag coefficient, and consequently for $\alpha=4.0^{\circ}$ and higher angles of attack the lift-to-drag ratio for the membrane blade is higher compared with the conventional rigid blade. While having a higher lift coefficient and lift-to-drag ratio is not desired for a stall-controlled turbine like the NASA-Ames Phase VI rotor, it should be mentioned that the purpose of the current study is to investigate the characteristics of the membrane blade concept and make a comparison between the membrane blade and a conventional blade. No conclusion could be made at this stage whether the concept should be utilized for pitch-controlled or stall-controlled turbines.

The cross section of the membrane blade varies along the span. Membrane deformation changes the cross section properties of the blade like the maximum camber and thickness. The original S809 airfoil has a maximum camber of about $1 \%$ at $82.3 \%$ chord position, but for the membrane blade, the maximum camber and its location changes with the angle of attack (Table 7). It is also shown in Fig. 19 that with the increase in the angle of attack the maximum camber increases as well, and in general the point of maximum camber moves slightly towards the leading edge of the blade.

The increase in the lift coefficient for the membrane blade could also be seen in the pressure coefficient distribution over the blade's surface. The pressure coefficient distribution over the middle span section of the second segment from the root is plotted in Fig. 20 and is compared with the pressure coefficient distribution of the rigid blade for $\alpha=6.0^{\circ}$. The kink in the $c_{p}$ distribution is due to slope discontinuity at the point 
where the lower membrane is attached to the rigid leading edge mast.

\section{Conclusion}

Fluid-structure interaction simulation of a semi-flexible membrane blade configuration is done over a range of angles of attack. Two different fluid models were used: CFD simulation based on RANS equations and the vortex panel method. The panel method saves computation time while providing a good accuracy up to an angle of attack of $6^{\circ}$. This makes the panel method an appropriate tool for early design stages of the membrane blade where an extensive parameter study needs to be done. Its accuracy could be improved by coupling boundary layer models with it. Comparing the performance of the membrane blade with its representative rigid counterpart the following main observations are made:

1. A higher lift curve slope for the membrane blade is observed. Even though the membrane blade has a smaller lift coefficient at zero angle of attack than the rigid blade, due to the higher slope of the lift curve, the membrane blade shows a higher lift coefficient compared with the rigid blade.

2. With the increase in the angle of attack, the lift-to-drag ratio of the membrane blade becomes higher than that of the rigid blade.

3. The maximum camber and its location for a membrane blade depends on the angle of attack. The maximum camber of the membrane blade is higher than the rigid blade. With the increase in the angle of attack, there is a slight shift of the point of maximum camber toward the leading edge.

Acknowledgements. The support provided by the Deutsche Forschungsgemeinschaft (DFG) (BL 306/32-1) is gratefully acknowledged.

Edited by: G. J. W. van Bussel

Reviewed by: A. Vire and two anonymous referees

\section{References}

Abdulrahim, M., Garcia, H., and Lind, R.: Flight Characteristics of Shaping the Membrane Wing of a Micro Air Vehicle, J. Aircraft, 42, 131-137, doi:10.2514/1.4782, 2005.

Baumgärtner, D., Wolf, J., Rossi, R., Wüchner, R., and Dadvand, P.: Contribution to the Fluid-Structure Interaction Analysis of Ultra-Lightweight Structures using an Embedded Approach, International Center for Numerical Methods in Engineering, Vol. CIMNE Monograph M152, 2015.
Barbarino, S., Dettmer, W., and Friswell, M.: Morphing Trailing Edges with Shape Memory Alloy Rods, ICAST, 4-6 October, State College, PA, USA, 2010.

Barlas, A. K., Tibaldi, C., Zahle, F., and Madsen, H.: Aeroelastic Optimization of a $10 \mathrm{MW}$ Wind Turbine Blade with Active Trailing Edge Flaps, 34th Wind Energy Symposium, AIAA, doi:10.2514/6.2016-1262, 2016.

Bletzinger, K.-U. and Ramm, E.: A General Finite Element Approach to the form Finding of Tensile Structures by the Updated Reference Strategy, Int. J. Space Struct., 14, 131-145, 1999.

Blondeau, J., Richeson, J., and Pines, J. D.: Desing, development and testing of a morphing aspect ratio wing using an inflatable telescopic spar, 44th AIAA/ASME/ASCE/AHS/ASC Structures, Structural Dynamics, and Materials Conference, doi:10.2514/6.2003-1718, 2003.

Bottasso, C. L., Croceb, A., Gualdonib, F., and Montinarib, P.: Load mitigation for wind turbines by a passive aeroelastic device, J. Wind Eng. Ind. Aerod., 148, 57-69, doi:10.1016/j.jweia.2015.11.001, 2016.

Bowmann, J., Sanders, B., Cannon, B., Kudva, J., Joshi, S., and Weisshaar, T.: Development of Next Generation Morphing Aircraft Structures, 48th AIAA/ASME/ASCE/AHS/ASC Structures, Structural Dynamics, and Materials Conference, Honolulu, Hawaii, doi:10.2514/6.2007-1730, 2007.

Fink, M.: Full-scale Investigation of the Aerodynamic Characteristics of a Model Employing a Sailwing Concept, NASA-TN-D4062, 1967.

Fink, M.: Full-scale Investigation of the Aerodynamic Characteristics of a Sailwing of Aspect Ratio 5.9, NASA-TN-D-5047, 1969.

Hand, M., Simms, D., Fingersh, L., Jager, D., Cotrell, J., Schreck, S., and Larwood, S.: Unsteady aerodynamics experiment phase VI: wind tunnel test configurations and available data campaigns, Technical Report NREL/TP-500-29955, NREL, Golden, CO, USA, 2001.

Jasak, H.: Dynamic Mesh Handling in OpenFOAM, 48th AIAA Aerospace Sciences Meeting, Orlando, Florida, 2009.

Jasak, H. and Tukovic, Z.: Automatic mesh motion for unstructured finite volume method, T. FAMENA, 30, 1-18, 2007.

Katz, J. and Plotkin, A.: Low Speed Aerodynamics, Cambridge, Cambridge Univ. Press, 2008.

Kupzok, A. M.: Modeling the Interaction of Wind and Membrane Structures by Numerical Simulation, Technical University of Munich, 2009.

Levin O. and Shyy W.: Optimization of a flexible low Reynolds number airfoil, 39th Aerospace Sciences Meeting and Exhibit, Aerospace Sciences Meetings, doi:10.2514/6.2001-125, 2001.

Lian, Y. and Shyy, W.: Numerical Simulation of Membrane Wing Aerodynamics for Micro Air Vehicle Applications, J. Aircraft, 42, 865-873, doi:10.2514/1.5909, 2005.

Maughmer M. D.: Optimization and Characteristics of a Sailwing Windmill Rotor, Princeton University, 1976.

Maughmer, M. D.: A Comparison of the Aerodynamic Characteristics of Eight Sailwing Airfoil Sections, Proceedings of the 3rd International Symposium on the Science and Technology of Low Speed and Motorless Flight, Hampton, VA, USA, 155-176, 1979.

Menter, F. R.: Two-Equation Eddy-Viscosity Turbulence Models for Engineering Applications, AIAA J., 32, 1598-1605, doi:10.2514/3.12149, 1994. 
Ormiston, R.: Theoretical and Experimental Aerodynamics of the Sailwing, J. Aircraft, 8, 77-84, doi:10.2514/3.44232, 1971.

Otto, F. and Rasch, B.: Finding Form, Deutscher Werkbund Bayern, Edition A, Menges, 1995.

Patankar, S. V. and Spalding, D. B.: A calculation procedure for heat, mass and momentum transfer in three-dimensional parabolic flows, Int. J. Heat Mass Tran., 15, 1787-1806, doi:10.1016/0017-9310(72)90054-3, 1972.

Saeedi, M., Wüchner, R., and Bletzinger, K.-U.: Multi-fidelity Fluid-Structure Interaction Analysis of a Membrane Wing, 17th International Conference on Applied Aerodynamics and Aeromechanics, London, 169-176, 2015.

Schek, H.-J.: The force density method for form finding and computations of general networks, Comput. Method. Appl. M., 3, 115-134, doi:10.1016/0045-7825(74)90045-0, 1974.

Valasek, J.: Morphing Aerospace Vehicles and Structures, John Wiley, 2012.

Viré, A., Xiang, J., and Pain, C. C.: An immersed-shell method for modelling fluid-structure interactions, Philos. T. R. Soc., 373, 2035, doi:10.1098/rsta.2014.0085, 2015.
Wakefield, D. S.: Engineering analysis of tension structures: theory and practice, Eng. Struct., 21, 680-690, doi:10.1016/S01410296(98)00023-6, 1999.

Wang, T., Wüchner, R., Sicklinger, S., and Bletzinger, K.-U.: Assessment and improvement of mapping algorithms for nonmatching meshes and geometries in computational FSI, J. Comput. Mech., 57, 793-816, doi:10.1007/s00466-016-1262-6, 2016.

Waszak, R. M., Jenkins, N. L., and Ifju, P.: Stability and Control Properties of an Aeroelastic Fixed Wing Micro Aerial Vehicle, AIAA Atmospheric Flight Mechanics Conference and Exhibit, Guidance, Navigation, and Control and Co-located Conferences, Montreal, Canada, doi:10.2514/6.2001-4005, 2001.

Wüchner, R. and Bletzinger, K.-U.: Stress-adapted numerical form finding of pre-stressed surfaces by the updated reference strategy, Int. J. Numer. Method. Eng., 64, 143-166, doi:10.1002/nme.1344, 2005.

Wüchner, R., Kupzok, A., and Bletzinger, K.-U.: A framework for stabilized partitioned analysis of thin membranewind interaction, Int. J. Numer. Method. H., 54, 945-963, doi:10.1002/fld.1474, 2007. 